\title{
Các nhân tố tác động đến ý định sử dụng đồng hồ thông minh: Nghiên cứu tại Việt Nam
}

\section{Factors affecting the intention to use smart watches: Case of Vietnam}

\author{
Lê Quang Huy ${ }^{1 *}$, Đỗ Thị Dung ${ }^{1}$ \\ ${ }^{1}$ Trường Đại học Tài chính - Marketing, Việt Nam \\ *Tác giả liên hệ, Email: quanghuy@ufm.edu.vn
}

THÔNG TIN

DOI: $10.46223 / \mathrm{HCMCOUJS}$. econ.vi.17.1.65.2022

Ngày nhận: 04/05/2020

Ngày nhận lại: 29/03/2020

Duyệt đăng: 20/04/2020

\section{Tù khóa:}

đồng hồ thông minh; ý định sử dụng

Keywords:

smartwatch adoption; intention to use

\section{TÓM TẮT}

Nghiên cứu này nhằm khám phá các nhân tố tác động đến ý định sử dụng Đồng Hồ Thông Minh (ĐHTM) tại thị trường Việt Nam. Nghiên cứu áp dụng khung lý thuyết của mô hình TAM, trong đó bổ sung thêm đặc tính văn hóa tiêu dùng tại Việt Nam. Nghiên cứu sử dụng phương pháp phân tích nhân tố khẳng định CFA và mô hình cấu trúc tuyến tính $S E M$ để kiểm định và đo lường mô hình lý thuyết, trải qua quy trình nghiên cứu sơ bộ và chính thức, dữ liệu khảo sát chính thức gồm 342 người tiêu dùng. Kết quả nghiên cứu cho thấy nhận thức tính hữu dụng và nhận thức tính dễ sử dụng tác động đến thái độ và ý định sử dụng ĐHTM. Ngoài ra, đặc tính văn hóa cũng ảnh hưởng không nhỏ đến thái độ cũng như ý định sử dụng ĐHTM của người tiêu dùng Việt Nam.

\section{ABSTRACT}

Purpose - This research aims is to identify the key psychological determinants of smart watch adoption (i.e., Perceived affective quality (PAQ), Mobility (MB), Availability (AV), Trust) and develops an extended technology acceptance model (TAM) that integrates the findings into the original TAM constructs and adds the Vietnamese consumer cultural characteristics.

Design/methodology/approach - An online survey assessed the proposed psychological determinants of smart watch adoption. Confirmatory Factor Analysis (CFA) and structural equation modeling $(\mathrm{SEM})$ were conducted on collected data $(\mathrm{n}=$ 342) using the SPSS 20 and the AMOS 24 statistical software. The reliability and validity of the measurement assessing the proposed factor structure were examined via Crobach's alpha, EFA, CFA, while the strength and direction of the hypothesized causal paths among the constructs were analyzed via SEM.

Findings - The perceived usefulness and perceived ease of use together consumer cultural characteristics were found to be associated with the consumer's attitude and intention to use smartwatches. 


\section{Giới thiệu nghiên cứu}

Ngày nay, Đồng Hồ Thông Minh (ĐHTM) là một thiết bị công nghệ khá phổ biến trên thế giới. Theo Cecchinato, Cox, và Bird (2015), ĐHTM là một thiết bị đeo cổ tay với với khả năng vi tính hóa, có thể kết nối với các thiết bị khác thông qua kết nối không dây tầm ngắn; cung cấp thông báo cảnh báo; thu thập dữ liệu cá nhân thông qua một loạt các cảm biến và lưu trữ chúng và có tích hợp đồng hồ. Còn theo Christensson (2017), ĐHTM là một chiếc đồng hồ kỹ thuật số cung cấp nhiều tính năng khác ngoài xem thời gian, như theo dõi nhịp tim, theo dõi hoạt động và cung cấp lời nhắc trong suốt cả ngày cho người sử dụng. Giống như điện thoại thông minh, ĐHTM có màn hình cảm ứng, cho phép người sử dụng thực hiện các hành động bằng cách chạm hoặc vuốt trên màn hình. Kết quả khảo sát của Rakuten Insight (2018) về thị trường thiết bị công nghệ đeo (gồm ĐHTM) của 12 quốc gia tại Châu Á được công bố vào ngày 05 tháng 09 năm 2018 thì giá trị của thị trường này sẽ ước tính đạt con số khoảng 25 tỷ USD vào năm 2019.

Kết quả đã khám phá ra nhiều điều thú thú vị về thị trường thiết bị công nghệ thông minh tại Việt Nam như sau: người Việt Nam được đánh giá là có mức độ chấp nhận sử dụng ĐHTM cao thứ 03 , chỉ đứng sau Thái Lan và Trung Quốc, tỷ lệ này là $63 \%$, nhưng chỉ có $28 \%$ trong số người khảo sát cân nhắc sẽ sử dụng ĐHTM. Câu hỏi đặt ra là vậy nguyên nhân nào khiến thị trường Việt Nam còn do dự sử dụng ĐHTM.

Trên thế giới, đã có nhiều nghiên cứu khoa học về các yếu tố tác động đến ý định sử dụng thiết bị đeo công nghệ ở các thị trường như Trung Quốc, Thái Lan, Pháp, Hàn Quốc (Dutot, Bhatiasevi, \& Bellahom 2019; Kim \& Shin, 2015), nhưng chưa có nghiên cứu khoa học chuyên sâu nào về thị trường ĐHTM ở Việt Nam. Vì vậy, nhóm tác giả thực hiện nghiên cứu các nhân tố ảnh hưởng đến ý định sử dụng ĐHTM tại thị trường Việt Nam, trên cơ sở ứng dụng và mở rộng mô hình chấp nhận công nghệ TAM và có xét đến đặc tính văn hóa tiêu dùng của người tiêu dùng Việt Nam.

\section{Cơ sở khoa học và đề xuất giả thuyết nghiên cứu}

Theo nghĩa thông thường, ý định (Intention) là dự định hay kế hoạch mà con người ta sẽ làm việc gì đó. Theo Ajzen (2002), ý định là trung gian dẫn đến hành vi. Ý định là hành vi của con người được hướng dẫn bởi việc cân nhắc ba yếu tố niềm tin vào hành vi, niềm tin vào chuẩn mực chủ quan (tác động xã hội) và niềm tin vào sự kiểm soát. Các niềm tin này càng mạnh thì ý định để thực hiện hành vi của con người càng lớn (Ajzen, 2002). Ngoài ra, theo Elbeck và Tirtiroglu (2008), ý định mua là sự sẵn sàng của khách hàng trong việc mua sản phẩm. Dự đoán ý định mua là bước khởi đầu để dự báo hành vi mua thực tế của khách hàng (Howard \& Sheth, 1969). Có nhiều lý thuyết giải thích cho hành vi của người tiêu dùng.

Fishbein và Ajzen (1975) đưa ra Lý thuyết hành vi hợp lý (TRA), cho rằng con người thường cân nhắc kết quả của các hành động khác nhau trước khi thực hiện chúng và họ chọn thực hiện các hành động sẽ dẫn đến những kết quả họ mong muốn. Công cụ tốt nhất để phán đoán hành vi là ý định.

Theo lý thuyết TRA, ý định chịu ảnh hưởng bởi thái độ đối với hành vi và chuẩn chủ quan. Thái độ là cảm giác tích cực hoặc tiêu cực của cá nhân về việc thực hiện một hành vi nhất định. Thái độ miêu tả mức độ một cá nhân đánh giá kết quả của một hành động là tích cực hay tiêu cực. Chuẩn chủ quan là nhận thức của con người về việc phải ứng xử thế nào cho phù hợp với yêu cầu của xã hội.

Ajzen (1991) với Lý thuyết hành vi có kế hoạch (TPB), cho rằng ý định là nhân tố động cơ dẫn đến hành vi hay nói cách khác quyết định thực hiện hành vi là kết quả của ý định cá nhân đối với lựa chọn hành vi. Trên cơ sở, các lý thuyết về lựa chọn hành vi, ứng dụng trong lĩnh vực 
công nghệ, Davis $(1989,1993)$ đề xuất mô hình mới gọi là mô hình chấp nhận công nghệ (the Technology Acceptance Model- TAM).

Mô hình TAM cho rằng tính/nhận thức hữu dụng (Perceived Usefulness - PU) và tính/nhận thức dễ sử dụng (Perceived Ease of Use - PEOU) đối với công nghệ sẽ tác động đến thái độ và ý định sử dụng hay mua sản phẩm/dịch vụ công nghệ nào đó.

Trên cơ sở vận dụng mô hình TAM, tác giả đề xuất nghiên cứu các nhân tố tác động đến ý định sử dụng ĐHTM bao gồm: tính hữu dụng, tính dễ sử dụng, thái độ và ý định lựa chọn. Từ đó, các giả thuyết ban đầu được đề xuất như sau:

H1: Tính dễ sử dụng sẽ tác động tích cưc đến tính hũu dụng trong nhận thức của người sủ dụng đối với ĐHTM

\section{H2: Tính dễ sử dụng sẽ tác động tích cực đến thái độ của người sủ dụng đối với ĐHTM}

H3a: Tính hữu dụng sẽ tác động tích cục đến thái độ của người sủ dụng đối với ĐHTM

H3b: Tính hũu dụng sẽ tác động tích cưcc đến ý định sử dụng ĐHTM

H4: Thái độ sẽ tác động tích cực đến ý định sử dụng ĐHTM

Russell (2003) lập luận rằng ảnh hưởng là một khía cạnh cơ bản và phổ quát của con người trong tất cả các sự kiện đầy cảm xúc, đối với các đối tượng, địa điểm. Chất lượng ảnh hưởng đến cảm nhận (Chất lượng cảm nhận - Affective Perceived Qualitive) là mức độ mà người dùng tin rằng một kích thích có thể thay đổi sự ảnh hưởng cốt lõi. Trong các nhiều nghiên cứu về công nghệ cũng như ĐHTM, chất lượng cảm nhận cũng được khẳng định có tác động đến tính hữu dụng và tính dễ sử dụng (Dutot et al., 2019; Kim \& Shin, 2015). Từ đó, giả thuyết H5a và H5b được phát biểu như sau:

H5a: Chất lự̛ng cảm nhận sẽ có tác dụng tích cục đối với tính hũu dụng của ĐHTM

H5b: Chất lương cảm nhận sẽ có tác dụng tích cực đến tính dễ sủ dụng của ĐHTM

Thế mạnh của thiết bị đeo công nghệ là tính năng di động (Mobility) đầy đủ cho người dùng. Theo Seppälä và Alamäki (2003), khả năng di động đề cập đến ba yếu tố: tiện lợi, phương tiện và trực tiếp. Ngoài ra, theo Shin (2012), MB cho phép người dùng truy cập thông tin mà không bị hạn chế về thời gian hoặc không gian. Kim và Shin (2015) cho rằng tính năng di động loại bỏ các ràng buộc trong một không gian cố định và đề cập đến mức độ mà người dùng tin rằng họ có thể điều hướng tự do với các thiết bị của họ ở các địa điểm khác nhau và trong thời gian vận chuyển. Tính năng di động thường liên kết với tính năng sẵn có. Tính năng sẵn có (Availabitity) được xác định là mức độ mà người dùng tin rằng thiết bị cung cấp cho họ kết nối thời gian thực với thông tin và dịch vụ (Kim \& Shin, 2015; Shin, 2012). Tính năng sẵn có cũng được khẳng định có mối quan hệ tích cực với tính dễ sử dụng của ĐHTM (Dutot et al., 2019). Theo đó, nhóm tác giả đề xuất giả thuyết tiếp theo sau:

H6a. Tỉnh di động sẽ có tác động tích cực đến nhận thức nhận thức tính hữu dụng của ĐHTM

H6b. Tính di động sẽ có tác động tích cưc đến nhận thức tính dễ sư dụng của ĐHTM

H7a. Tính sã̃n có sẽ có tác động tích cực đến nhận thức tính hữu dụng của ĐHTM

H7b. Tính sã̃n có sẽ có tác động tích cực đến nhận thức tính dễ sủ dụng của ĐHTM

Tính tin cậy hay niềm tin (Trust) đối với hãng sản xuất là một trong những vấn đề lớn nhất cũng như là thách thức cản trở việc sử dụng những sản phẩm công nghệ mới đặc biệt là 
những sản phẩm liên quan đến việc sử dụng và chia sẻ dữ liệu. Bởi vì khi ĐHTM được đeo ở cổ tay nó thu thập và lưu trữ dữ liệu cá nhân, quyền riêng tư của người dùng có thể bị đe dọa chẳng hạn như hệ thống thanh toán di động và thương mại điện tử (Aljifri, Pons, \& Collins, 2003; Dutot, 2015; Srivastava, Chandra, \& Theng, 2010). Trong khi đó người dùng không có quyền kiểm soát những gì các nhà cung cấp dịch vụ hoặc công ty sản xuất có thể làm với dữ liệu của họ. Mối tương quan giữa tính tin cậy và tính hữu dụng được xác định là tích cực (Dutot et al., 2019; Gefen, Karahanna, \& Straub, 2003), tương tự, tính tin cậy cũng được khám phá có mối quan hệ tích cực với tính dễ sử dụng (Dutot, 2015; Dutot et al., 2019; Reid \& Yair, 2008). Ngoài ra, nếu mang theo ĐHTM với tính năng sẵn có nhưng không tin cậy cũng sẽ giảm mức độ nhận thức tích cực đối với thiết bị này. Vì thế, giả thuyết kế tiếp được phát biểu như sau:

H8a: Tính tin cậy sẽ có tác động tích cực đến nhận thức tính hũu dụng của ĐHTM

H8b: Tính tin cậy sẽ có tác động tích cực đến tính dễ sử dụng của ĐHTM

H8c: Tính tin cậy sẽ có tác động tích cực đến tính sã̃n có của ĐHTM

Theo Bajarin (2014), mọi người mua ĐHTM không chỉ là để xem giờ mà còn vì thời trang. Yếu tố văn hóa cũng đã được khẳng định mối quan hệ tích cực đến thái độ đối với ĐHTM của Hàn Quốc (Kim \& Shin, 2015). Ngoài ra, nhóm tác giả cho rằng, những nước có nền văn hóa theo chủ nghĩa tập thể cao gồm các nước Châu Á trong đó có Việt Nam (Hofstede, 1983) có thể thái độ đối với sản phẩm nào đó không tốt nhưng người tiêu dùng vẫn có ý định mua sản phẩm vì theo số đông, trào lưu. Do vậy, giả thuyết H9a và $\mathrm{H} 9 \mathrm{~b}$ được phát biểu như sau:

H9a: Đặc tính văn hóa sẽ có tác động tích cưc đến thái độ của người sư dụng đối với ĐHTM

H9b: Đặc tính văn hóa sẽ có tác động tích cực đến ý định sủ dụng đối với ĐHTM

Mô hình giả thuyết nghiên cứu được đề xuất như hình dưới đây:

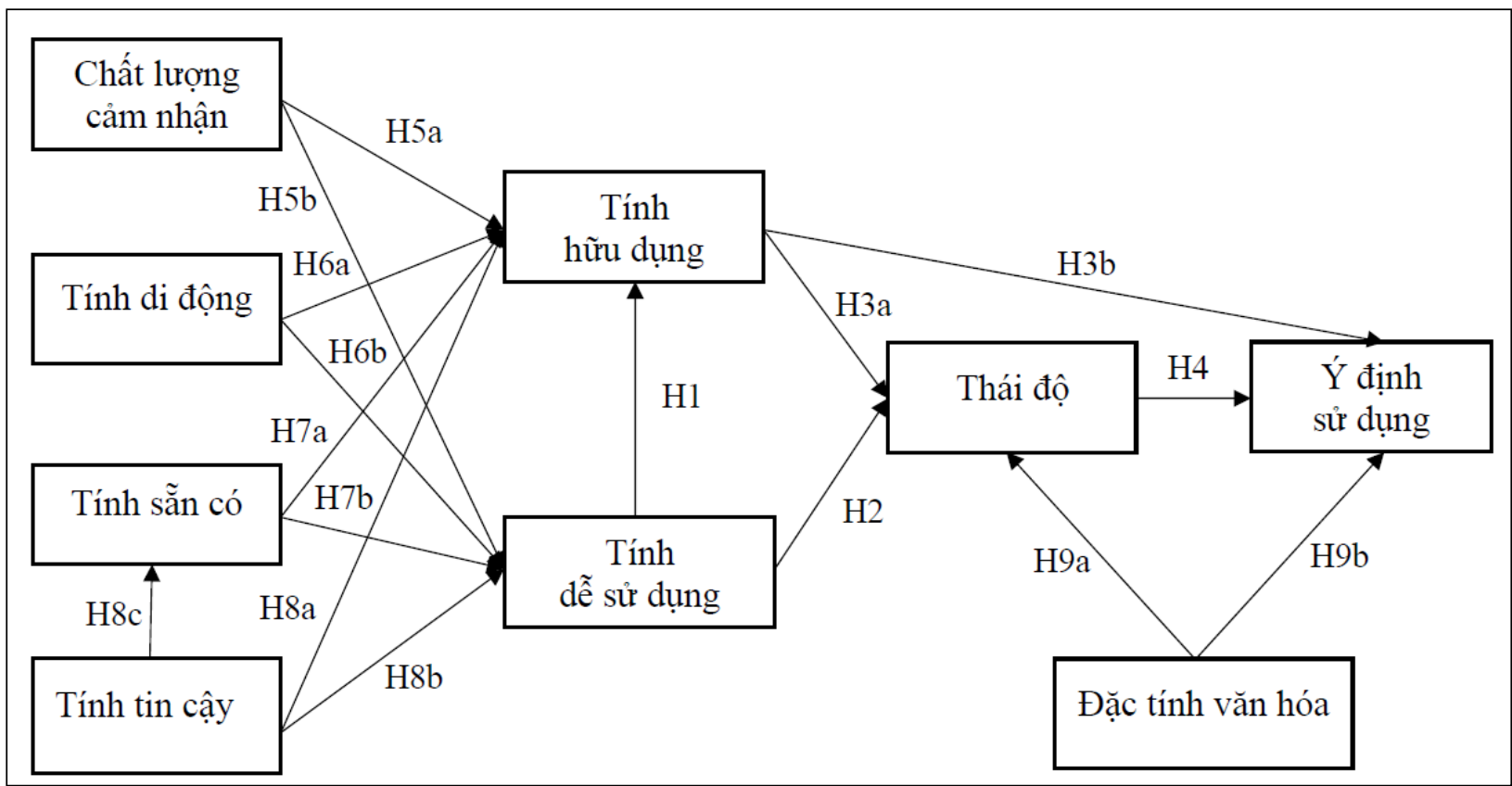

Hình 1. Mô hình giả thuyết nghiên cứu

Nguồn: Nhóm tác giả 


\section{Thiết kế nghiên cứu}

\subsection{Quy trình nghiên cúu}

- Quy trình nghiên cứu được thực hiện thông qua 02 bước chính: sơ bộ và chính thức. Trong sơ bộ định tính nhằm khám phá và điều chỉnh thang đo cho phù hợp với bối cảnh nghiên cứu của Việt Nam, chúng tôi tiến hành phỏng vấn bán cấu trúc với 04 nhóm tập trung trực tuyến bằng phần mềm Zoom. Trong đó, 03 nhóm gồm 140 sinh viên Trường Đại học Tài chính Marketing, năm thứ hai, ba, tư. Một nhóm là đối tượng đã đi làm gồm 10 cựu sinh viên Trường Đại học Tài chính Marketing, đã tốt nghiệp từ năm 2012 đến 2017 (thời gian thu thập dữ liệu: 17 - 22/02/2020). Tiếp theo, chúng tôi tiến hành khảo sát sơ bộ định lượng thông qua công cụ Google Form, thu về 174 phiếu khảo sát, gạn lọc được 150 phiếu đạt yêu cầu đưa vào phân tích (thời gian thu thập dữ liệu: 24 - 29/02/2020). Ở bước này, chúng tôi đánh giá độ tin cậy, giá trị thang đo thông qua phân tích Cronbach's alpha và phân tích nhân tố khám phá EFA;

- Ở bước nghiên cứu chính thức, chúng tôi tiến hành khảo sát thông qua công cụ Google Form thu về 380 phiếu khảo sát, gạn lọc được 342 phiếu đạt yêu cầu (thời gian thu thập dữ liệu: 08 - 14/03/2020). Sau đó, chúng tôi thực hiện đánh giá tin cậy thang đo, giá trị thang đo với EFA, phân tích nhân tố khẳng định CFA và kiểm định giả thuyết cũng như đánh giá mô hình lý thuyết thông qua SEM;

- Tiêu chí chọn mẫu khảo sát và cách thức gạn lọc đối tượng khảo sát: Mẫu nghiên cứu được khảo sát theo phương pháp thuận tiện. Đối tượng khảo sát là những người có ý định sử dụng đồng hồ thông minh. Kết quả khảo sát sau khi gạn lọc các bản câu hỏi thiếu nhiều thông tin, hoặc có trên một trả lời cho một câu hỏi, hoặc có cơ sở để xác định không đáng tin cậy (chẳng hạn: chọn cùng một, hoặc hai mức độ trả lời cho tất cả các câu hỏi) được nhập vào ma trận dữ liệu trên phần mềm SPSS 20 và được làm sạch sau đó, trước khi sử dụng để thống kê và phân tích dữ liệu.

\subsection{Phương pháp đo lưòng}

Các thang đo được dựa vào các nghiên cứu trước đó và phỏng vấn nhóm tập trung trong bước nghiên cứu định tính để điều chỉnh thang đo cho phù hợp với ngữ cảnh Việt Nam. Thang đo tính hữu dụng ban đầu bao gồm bốn biến quan sát theo mô hình TAM của Davis $(1989,1993)$ và thang đo của Dutot và các cộng sự (2019). Tuy nhiên qua nghiên cứu định tính sơ bộ, nhóm tác giả khám phá thêm hai biến mới bổ sung vào thang đo tính hữu dụng gồm "ĐHTM có thể giúp tôi giải cứu và cảnh báo trong tình huống nguy hiểm" và "ĐHTM có thể giúp tôi theo dõi sức khoẻ liên tục.” Thang đo tính dễ sử dụng (04 biến quan sát), Thang đo thái độ (04 biến quan sát) và thang đo ý định sử dụng (03 biến quan sát) được trích xuất từ mô hình TAM của Davis $(1989,1993)$ và thang đo của Dutot và các cộng sự (2019). Thang đo chất lượng cảm nhận (03 biến quan sát) dựa vào thang đo của Kim và Shin (2015). Thang đo tính di động (03 biến quan sát) dựa vào thang đo của Kim và Shin (2015) và Thang đo tính sẵn có (04 biến quan sát) dựa vào thang đo của Dutot (2015), Dutot và các cộng sự (2019), Thang đo tính tin cậy (04 biến quan sát) dựa vào thang đo của Dutot (2015), Dutot và các cộng sự (2019). Thang đo đặc tính văn hóa (04 biến quan sát) dựa vào thang đo của Kim và Shin (2015) và bối cảnh nghiên cứu ở Việt Nam.

\section{Kết quả nghiên cứu}

\subsection{Kết quả nghiên cứu sơ bộ định lự̣ng}

Kết quả nghiên cứu định lượng sơ bộ với mẫu nghiên cứu 150 quan sát. Qua đó, 05 biến quan sát độc lập được loại khỏi mô hình nghiên cứu, trong đó hai biến không đạt yêu cầu hệ số tương quan biến tổng trong phân tích Cronbach's alpha, ba biến không đạt được giá trị thang đo 
trong phân tích EFA. Sau hai lần đánh giá, độ tin cậy thang đo, hệ số Cronbach's Alpha chuẩn hóa đạt 0.923 và giá trị thang đo với hệ số KMO đạt 0.835 (đạt yêu cầu), còn lại tập 30 biến quan sát được đưa vào thực hiện ở nghiên cứu chính thức định lượng.

\subsection{Kết quả nghiên cứu chính thức}

4.2.1. Đặc điểm mẫu nghiên cứu chính thức và kết quả thống kê mô tả các biến quan sát

Mẫu khảo sát chính thức sau khi gạn lọc bao gồm 342 quan sát, trong đó đối tượng có giới tính nữ chiếm $71.1 \%$, nam chiếm $28.9 \%$. Độ tuổi khảo sát chủ yếu tập trung vào giới trẻ, có độ tuổi từ 18 - 35 chiếm $91.2 \%$. Về thu nhập, đối tượng có thu nhập chiếm khoảng $52 \%$ trong tổng số đối tượng khảo sát. Tương tự, các đặc điểm khác về mẫu nghiên cứu được mô tả như Bảng 1. Mặc dù, nghiên cứu sử dụng phương pháp chọn mẫu thuận tiện, nhưng kết quả thống kê mô tả mẫu tương đối phù hợp với kết quả khảo sát đặc điểm tiêu dùng sản phẩm công nghệ (ĐHTM) của Việt Nam của một số công ty nghiên cứu thị trường (Rakuten Insight, 2018).

\section{Bảng 1}

Kết quả thống kê đặc điểm nhân khẩu học mẫu nghiên cứu chính thức

\begin{tabular}{|c|c|c|}
\hline & & $\mathbf{N}(\%)$ \\
\hline \multirow{2}{*}{ Giới tính } & Nữ & $243(71.1 \%)$ \\
\hline & Nam & $99(28.9 \%)$ \\
\hline \multirow{3}{*}{ Độ tuổi } & 18 tuổi - 35 tuổi & $312(91.2 \%)$ \\
\hline & 36 tuổi - 55 tuổi & $27(7.9 \%)$ \\
\hline & Trên 55 & $3(0.9 \%)$ \\
\hline \multirow{3}{*}{ Học vấn } & Đại học & $292(85.4 \%)$ \\
\hline & Trên đại học & $22(6.4 \%)$ \\
\hline & Khác & $28(8.2 \%)$ \\
\hline \multirow{4}{*}{ Ngành nghề } & Sinh viên & $208(60.8 \%)$ \\
\hline & Nhân viên văn phòng & $70(20.5 \%)$ \\
\hline & Quản lý & $59(17.3 \%)$ \\
\hline & Công nhân & $5(1.5 \%)$ \\
\hline \multirow{5}{*}{ Thu nhập } & Chưa có thu nhập & $165(48.2 \%)$ \\
\hline & Dưới 7,000,000 đồng & $65(19 \%)$ \\
\hline & Trên 7,000,000 đến dưới 11,000,000 đồng & $41(12 \%)$ \\
\hline & Trên $11,000,000$ đến dưới $25,000,000$ đồng & $63(18.4 \%)$ \\
\hline & Trên $25,000,000$ đồng & $8(2.3 \%)$ \\
\hline
\end{tabular}

Nguồn: Kết quả xử lý dữ liệu khảo sát

Theo kết quả thống kê mô tả như Bảng 2, giá trị trung bình của tất cả các biến quan sát xoay quanh 03 , nằm trong khoảng từ $03-03.5 / 5$. Mặt khác giá trị Độ lệch và Độ nhọn nhỏ hơn 0 , nằm trong khoảng -01 đến 0 , cho thấy chuỗi dữ liệu nghiên cứu có đồ thị phân phối lệch trái 
và mức độ tập trung đều về 02 bên nên có sự biến động nhẹ giữa giá trị trung bình, giá trị lớn nhất, giá trị nhỏ nhất và độ lệch chuẩn.

\section{Bảng 2}

Kết quả thống kê mô tả các biến quan sát

\begin{tabular}{|c|c|c|c|c|c|c|c|c|c|}
\hline & \multirow{2}{*}{$\begin{array}{c}\text { Số quan } \\
\text { sát }\end{array}$} & \multirow[b]{2}{*}{ Tối thiểu } & \multirow[b]{2}{*}{ Tối đa } & \multirow[b]{2}{*}{ Trị trung bình } & \multirow[b]{2}{*}{ Phương sai } & \multicolumn{2}{|c|}{ Độ lệch } & \multicolumn{2}{|c|}{ Độ nhọn } \\
\hline & & & & & & Giá trị & $\begin{array}{l}\text { Sai lệch } \\
\text { chuẩn }\end{array}$ & Giá trị & $\begin{array}{l}\text { Sai lệch } \\
\text { chuẩn }\end{array}$ \\
\hline THD1 & 342 & 1.00 & 5.00 & 3.2018 & 1.487 & -.206 & .132 & \begin{tabular}{|c|}
-.912 \\
\end{tabular} & .263 \\
\hline THD2 & 342 & 1.00 & 5.00 & 3.2193 & 1.427 & -.264 & .132 & -.822 & .263 \\
\hline THD3 & 342 & 1.00 & 5.00 & 3.0058 & 1.548 & -.112 & .132 & -.918 & .263 \\
\hline THD4 & 342 & 1.00 & 5.00 & 3.2661 & 1.410 & -.284 & .132 & -.897 & .263 \\
\hline TDSD1 & 342 & 1.00 & 5.00 & 3.1930 & 1.482 & -.217 & .132 & -.869 & .263 \\
\hline TDSD2 & 342 & 1.00 & 5.00 & 3.0789 & 1.551 & -.150 & .132 & -1.014 & .263 \\
\hline TDSD3 & 342 & 1.00 & 5.00 & 3.3070 & 1.556 & -.306 & .132 & \begin{tabular}{l|l|}
-.893 \\
\end{tabular} & .263 \\
\hline CLCN1 & 342 & 1.00 & 5.00 & 3.2251 & 1.237 & \begin{tabular}{l|l|}
-248 \\
\end{tabular} & .132 & -.719 & .263 \\
\hline CLCN2 & 342 & 1.00 & 5.00 & 3.1550 & 1.586 & -.180 & .132 & -.997 & .263 \\
\hline CLCN3 & 342 & 1.00 & 5.00 & 3.2018 & 1.440 & -.230 & .132 & -.831 & .263 \\
\hline TDD1 & 342 & 1.00 & 5.00 & 3.1813 & 1.457 & -.121 & .132 & -.909 & .263 \\
\hline TDD2 & 342 & 1.00 & 5.00 & 3.0380 & 1.438 & -.145 & .132 & -.938 & .263 \\
\hline TDD3 & 342 & 1.00 & 5.00 & 3.0556 & 1.654 & -.071 & .132 & -1.100 & .263 \\
\hline TSC1 & 342 & 1.00 & 5.00 & 3.1696 & 1.478 & -.191 & .132 & -.927 & .263 \\
\hline TSC2 & 342 & 1.00 & 5.00 & 3.1111 & 1.425 & -.070 & .132 & -.850 & .263 \\
\hline TSC3 & 342 & 1.00 & 5.00 & 3.0731 & 1.546 & -204 & .132 & -.915 & .263 \\
\hline TTC1 & 342 & 1.00 & 5.00 & 3.2836 & 1.400 & $\mid-287$ & .132 & -.836 & .263 \\
\hline TTC2 & 342 & 1.00 & 5.00 & 3.2427 & 1.322 & \begin{tabular}{|c|}
-287 \\
\end{tabular} & .132 & \begin{tabular}{|c|}
-.673 \\
\end{tabular} & .263 \\
\hline TTC3 & 342 & 1.00 & 5.00 & 3.2807 & 1.293 & $\begin{array}{l}-.327 \\
\end{array}$ & .132 & $\begin{array}{l}-699 \\
\end{array}$ & .263 \\
\hline TTC4 & 342 & 1.00 & 5.00 & 3.2661 & 1.609 & \begin{tabular}{l|}
-.285 \\
\end{tabular} & .132 & \begin{tabular}{l|}
-.956 \\
\end{tabular} & .263 \\
\hline $\mathrm{VH} 1$ & 342 & 1.00 & 5.00 & 3.0497 & 1.467 & $\begin{array}{l}-165 \\
\end{array}$ & .132 & -.933 & .263 \\
\hline $\mathrm{VH} 2$ & 342 & 1.00 & 5.00 & 3.2281 & 1.438 & \begin{tabular}{|l|}
-191 \\
\end{tabular} & .132 & \begin{tabular}{|c|}
-861 \\
\end{tabular} & .263 \\
\hline $\mathrm{VH} 3$ & 342 & 1.00 & 5.00 & 3.2719 & 1.389 & \begin{tabular}{l|}
-282 \\
\end{tabular} & .132 & \begin{tabular}{|c|}
-.687 \\
\end{tabular} & .263 \\
\hline TD1 & 342 & 1.00 & 5.00 & 3.2193 & 1.216 & \begin{tabular}{|c|}
-.337 \\
\end{tabular} & .132 & \begin{tabular}{|c|}
-.577 \\
\end{tabular} & .263 \\
\hline TD2 & 342 & 1.00 & 5.00 & 3.2135 & 1.611 & -.303 & .132 & -.965 & .263 \\
\hline TD3 & 342 & 1.00 & 5.00 & 3.1140 & 1.439 & -.180 & .132 & -.880 & .263 \\
\hline TD4 & 342 & 1.00 & 5.00 & 3.2427 & 1.463 & \begin{tabular}{l|}
-295 \\
\end{tabular} & .132 & -.804 & .263 \\
\hline YD1 & 342 & 1.00 & 5.00 & 3.2982 & 1.406 & \begin{tabular}{|l|}
-.319 \\
\end{tabular} & .132 & \begin{tabular}{l|l|}
-.802 \\
\end{tabular} & .263 \\
\hline YD2 & 342 & 1.00 & 5.00 & 3.2690 & 1.652 & -.363 & .132 & \begin{tabular}{|c|}
-.901 \\
\end{tabular} & .263 \\
\hline YD3 & 342 & 1.00 & 5.00 & 3.2368 & 1.618 & -.212 & .132 & -.980 & .263 \\
\hline
\end{tabular}

Nguồn: Kết quả xử lý dữ liệu khảo sát

\subsection{2. Đánh giá độ tin câyy và giá trị của thang đo}

Kết quả chung đánh giá độ tin cậy thang đo, cho biết hệ số Cronbach's Alpha của tập 30 biến quan sát là 0.886 , và hệ số tương quan biến tổng của tất cả các biến đạt yêu cầu $(>0.3)$ Tiếp theo, đánh giá giá trị thang đo với EFA, một biến bị loại do không đạt được hội tụ (THD3), còn lại 29 biến quan sát đạt ý nghĩa thống kê và lập thành 09 nhóm nhân tố với hệ số KMO đạt 0.866 và Eigenvalue với tổng phương sai trích là $64.34 \%$. 


\section{Bảng 3}

Thống kê hệ số tương quan biến tổng

\begin{tabular}{|c|c|c|c|c|c|}
\hline & $\begin{array}{l}\text { Scale Mean if } \\
\text { Item Deleted }\end{array}$ & $\begin{array}{l}\text { Scale Variance } \\
\text { if Item Deleted }\end{array}$ & $\begin{array}{l}\text { Corrected } \\
\text { Item-Total } \\
\text { Correlation }\end{array}$ & $\begin{array}{c}\text { Squared } \\
\text { Multiple } \\
\text { Correlation }\end{array}$ & $\begin{array}{l}\text { Cronbach's } \\
\text { Alpha if Item } \\
\text { Deleted }\end{array}$ \\
\hline THD1 & 92.4971 & 282.790 & .507 & .534 & .880 \\
\hline THD2 & 92.4795 & 286.121 & .434 & .465 & .881 \\
\hline THD3 & 92.6930 & 290.577 & .305 & .201 & .884 \\
\hline THD4 & 92.4327 & 283.777 & .497 & .546 & .880 \\
\hline TDSD1 & 92.5058 & 287.295 & .395 & .352 & .882 \\
\hline TDSD2 & 92.6199 & 287.098 & .389 & .379 & .882 \\
\hline TDSD3 & 92.3918 & 285.101 & .437 & .410 & .881 \\
\hline CLCN1 & 92.4737 & 288.285 & .412 & .306 & .882 \\
\hline CLCN2 & 92.5439 & 285.709 & .418 & .352 & .882 \\
\hline CLCN3 & 92.4971 & 287.330 & .401 & .357 & .882 \\
\hline TDD1 & 92.5175 & 286.837 & .411 & .317 & .882 \\
\hline TDD2 & 92.6608 & 284.964 & .461 & .380 & .881 \\
\hline TDD3 & 92.6433 & 286.295 & .394 & .326 & .882 \\
\hline TSC1 & 92.5292 & 284.467 & .466 & .334 & .881 \\
\hline $\mathrm{TSC} 2$ & 92.5877 & 285.592 & .448 & .376 & .881 \\
\hline TSC3 & 92.6257 & 286.258 & .411 & .344 & .882 \\
\hline TBM1 & 92.4152 & 289.528 & .351 & .300 & .883 \\
\hline TBM2 & 92.4561 & 290.900 & .328 & .423 & .884 \\
\hline TBM3 & 92.4181 & 287.710 & .417 & .355 & .882 \\
\hline TBM4 & 92.4327 & 287.847 & .363 & .463 & .883 \\
\hline VH1 & 92.6491 & 282.252 & .525 & .436 & .879 \\
\hline $\mathrm{VH} 2$ & 92.4708 & 283.892 & .489 & .442 & .880 \\
\hline VH3 & 92.4269 & 284.011 & .495 & .409 & .880 \\
\hline TD1 & 92.4795 & 287.928 & .426 & .331 & .882 \\
\hline TD2 & 92.4854 & 284.597 & .441 & .410 & .881 \\
\hline TD3 & 92.5848 & 285.980 & .435 & .386 & .881 \\
\hline TD4 & 92.4561 & 286.149 & .427 & .364 & .882 \\
\hline YD1 & 92.4006 & 284.176 & .488 & .415 & .880 \\
\hline YD2 & 92.4298 & 286.791 & .382 & .307 & .883 \\
\hline YD3 & 92.4620 & 286.003 & .406 & .299 & .882 \\
\hline
\end{tabular}

Nguồn: Kết quả xử lý dữ liệu khảo sát 


\section{Bảng 4}

Kết quả ma trận xoay nhân tố lần 2

\begin{tabular}{|c|c|c|c|c|c|c|c|c|c|}
\hline & \multicolumn{9}{|c|}{ Component } \\
\hline & 1 & 2 & 3 & 4 & 5 & 6 & 7 & 8 & 9 \\
\hline THD1 & & & .791 & & & & & & \\
\hline THD2 & & & .817 & & & & & & \\
\hline THD4 & & & .819 & & & & & & \\
\hline TDSD1 & & & & & .743 & & & & \\
\hline TDSD2 & & & & & .778 & & & & \\
\hline TDSD3 & & & & & .798 & & & & \\
\hline CLCN1 & & & & & & & & & .707 \\
\hline CLCN2 & & & & & & & & & .728 \\
\hline CLCN3 & & & & & & & & & .747 \\
\hline TDD1 & & & & & & & & .718 & \\
\hline TDD2 & & & & & & & & .689 & \\
\hline TDD3 & & & & & & & & .777 & \\
\hline TSC1 & & & & & & & .685 & & \\
\hline TSC2 & & & & & & & .787 & & \\
\hline TSC3 & & & & & & & .726 & & \\
\hline TBM1 & & .639 & & & & & & & \\
\hline TBM2 & & .794 & & & & & & & \\
\hline TBM3 & & .689 & & & & & & & \\
\hline TBM4 & & .771 & & & & & & & \\
\hline VH1 & & & & .707 & & & & & \\
\hline VH2 & & & & .791 & & & & & \\
\hline VH3 & & & & .712 & & & & & \\
\hline TD1 & .698 & & & & & & & & \\
\hline TD2 & .726 & & & & & & & & \\
\hline TD3 & .726 & & & & & & & & \\
\hline TD4 & .690 & & & & & & & & \\
\hline YD1 & & & & & & .737 & & & \\
\hline YD2 & & & & & & .748 & & & \\
\hline YD3 & & & & & & .689 & & & \\
\hline
\end{tabular}

Extraction Method: Principal Component Analysis.

Rotation Method: Varimax with Kaiser Normalization.

a. Rotation converged in 7 iterations.

Nguồn: Kết quả xử lý dữ liệu khảo sát 


\subsubsection{Phân tích kết quả khẳng định thang đo với $C F A$}

Với phương pháp EFA, giúp cho nhà nghiên cứu khám phá dữ liệu nghiên cứu sẽ bao gồm bao nhiêu nhóm nhân tố cần thiết tốt nhất để đại diện cho dữ liệu. Tuy nhiên, đặc trưng của EFA là các nhóm nhân tố được rút ra từ kết quả thống kê chứ không phải từ lý thuyết (Hair, Black, Babin, \& Anderson, 2009, 2014). Nên để kiểm định xem kết quả các nhóm nhân tố phù hợp với lý thuyết hay không, các nhà nghiên cứu sử dụng một trong những phương pháp đó là CFA (Hair et al., 2009, 2014).

Trong phân tích CFA, một mô hình được gọi là phù hợp với dữ liệu thị trường nếu phép kiểm định Chi - bình phương có giá trị $\mathrm{p}$ lớn hơn $5 \%$, tuy nhiên Chi - bình phương lại phụ thuộc vào kích thước mẫu nên thay vào đó mô hình được đánh giá phù hợp thông qua: chỉ số TLI (Tucker \& Lewis, 1973) và chỉ số thích hợp so sánh CFI (Comparative Fit Index) có giá trị từ 0.9 đến 1.0, chỉ số Chi - bình phương điều chỉnh theo bậc tự do CMIN/df có giá trị nhỏ hơn 02, chỉ số RMSEA (Root mean square error approximation) có giá trị nhỏ hơn 0.8 (Hair et al., 2009, 2014; Nguyen \& Nguyen, 2011).

Ngoài ra để kiểm định giá trị thang đo, người ta đánh giá thông qua các chỉ tiêu như sau: (1) Hệ số tin cậy tổng hợp (Composite reliability), (2) Tổng phương sai trích (Variance extracted), (3) Tính đơn hướng (Unidimensionality), (4) Giá trị hội tụ (Convergent validity), (5) Giá trị phân biệt (Discriminant validity) và (6) Giá trị liên hệ lý thuyết (Nomological validity). Trong đó, các chỉ tiêu từ (1) đến (5) được đánh giá trong mô hình thang đo (thông qua phân tích CFA), chỉ tiêu (6) được đánh giá trong mô hình lý thuyết (thông qua phân tích cấu trúc tuyến tính SEM) (Anderson \& Gerbing, 1988; Nguyen \& Nguyen, 2011). Theo Hair và cộng sự (2009, 2014), trong phân tích CFA, giá trị hội tụ đạt yêu cầu khi có hệ số tải nhân tố (Factor loadings) lớn hơn 0.5 , tốt hơn nữa là 0.7 hoặc cao hơn; cùng với phương sai trích trung bình (Average variance extracted, viết tắt là $\mathrm{AVE}$ ) lớn hơn 0.5 ; và chỉ số độ tin cậy tổng hợp - (Construct/ Composite reliability), viết tắt là C.R) có giá trị 0.6 - 0.7 chấp nhận được, lớn hơn 0.7 càng tốt. Tuy nhiên, theo Fornell và Larcker (1981), nếu phương sai trích trung bình nhỏ hơn 0.5 thì chỉ số độ tin cậy tổng hợp C.R lớn hơn 0.6 là chấp nhận được.

Với kết quả CFA chuẩn hóa như Hình 2, giá trị CMIN/df là 1.042, chỉ số CFI đạt 0.997 , GFI đạt 0.935 , TLI là 0.996 , RMSEA đạt 0.008 , tất cả đều đạt yêu cầu. Ngoài ra, tất cả các thang đo thành phần đều có trọng số ước lượng lớn hơn 0.5 ; thấp nhất là biến TTC1 thuộc thang đo TTC có trọng số 0.51 . Bên cạnh đó, giá trị tới hạn cao hơn rất nhiều so với 1.96 và giá trị $\mathrm{P}$ ở trong các bảng ước lượng ký hiệu $(* * *)$, nên kết luận rằng kết quả ước lượng đạt mức ý nghĩa thống kê. 


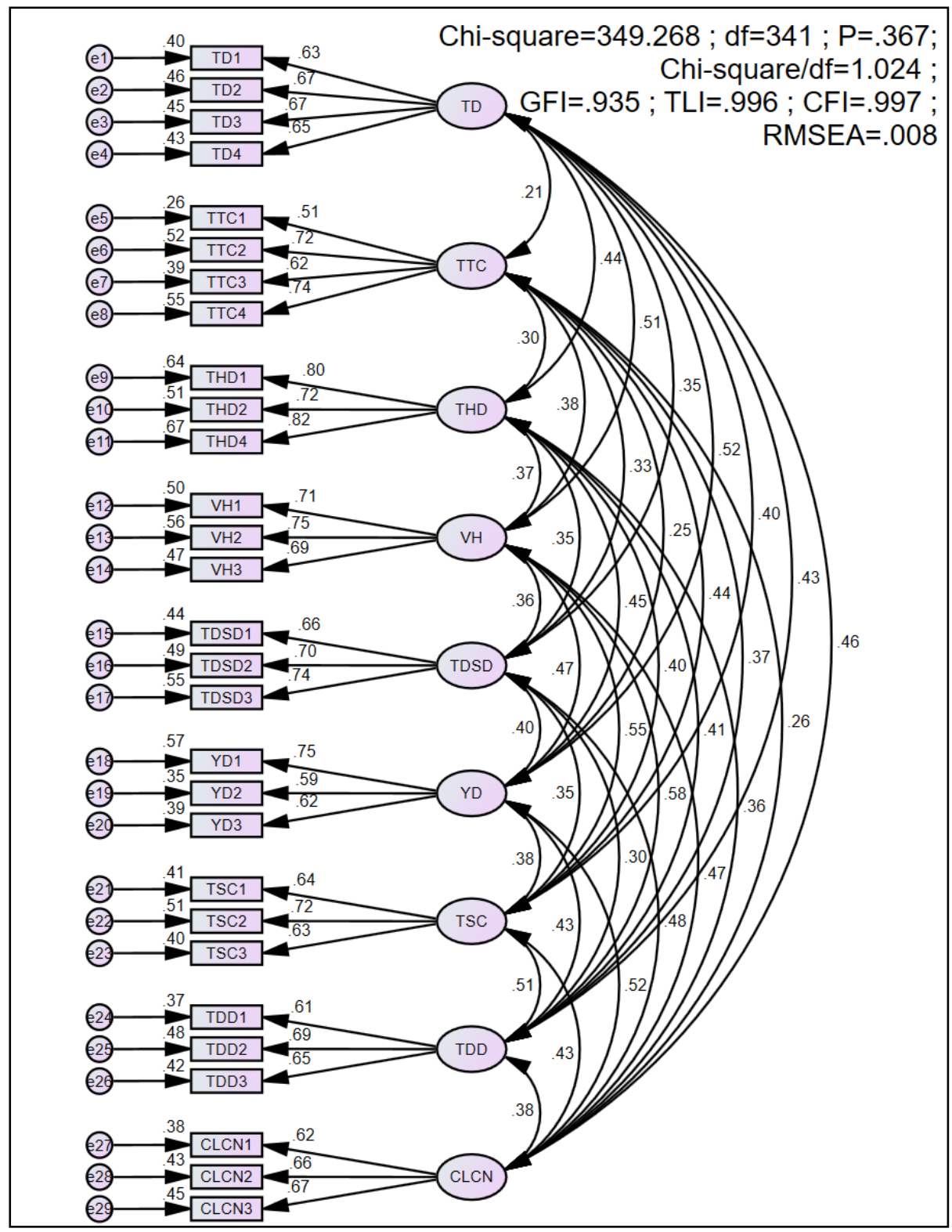

Hình 2. Kết quả CFA mô hình đo lường tới hạn (chuẩn hóa)

Nguồn: Kết quả xử lý dữ liệu khảo sát

Tiếp theo, kết quả phương sai trích của tất cả các thang đo đều $>40 \%$, thang đo THD có giá trị AVE lớn nhất với $60.9 \%$, mặt khác tất cả thang đo đều có chỉ số tin cậy tổng hợp $>60 \%$. (Bảng 5). Từ đó, khẳng định rằng tất cả các thang đo đều đạt được giá trị hội tụ.

\section{Bảng 5}

Kết quả ước lượng hệ số tin cậy tổng hợp và phương sai trích trung bình

\begin{tabular}{|c|c|c|c|c|c|c|c|c|c|c|c|c|c|}
\hline & CR & AVE & MSV & $\operatorname{MaxR}(H)$ & TD & TTC & THD & VH & TDSD & YD & TSC & TDD & CLCN \\
\hline TD & 0.753 & 0.433 & 0.268 & 0.754 & 0.658 & & & & & & & & \\
\hline TTC & 0.748 & $0.431 \mid$ & $0.193 \mid$ & 0.768 & $0.211^{* *}$ & 0.656 & & & & & & & \\
\hline THD & 0.823 & $|0.609|$ & 0.200 & 0.830 & $0.444 * * *$ & $0.303 * * *$ & 0.780 & & & & & & \\
\hline VH & 0.758 & 0.511 & 0.333 & 0.760 & $0.514 * * *$ & $0.379 * * *$ & $0.372 * * *$ & 0.715 & & & & & \\
\hline
\end{tabular}




\begin{tabular}{|c|c|c|c|c|c|c|c|c|c|c|c|c|c|}
\hline & CR & AVE & MSV & $\operatorname{MaxR}(H)$ & TD & TTC & THD & VH & TDSD & YD & TSC & TDD & CLCN \\
\hline TDSD & 0.743 & 0.492 & 0.230 & 0.747 & $0.355 * * *$ & $0.335 * * *$ & $0.347 * * *$ & $0.361 * * *$ & 0.701 & & & & \\
\hline YD & 0.695 & 0.435 & 0.268 & 0.713 & $0.518 * * *$ & $0.246 * *$ & $0.447 * * *$ & $0.470 * * *$ & $0.400 * * *$ & 0.659 & & & \\
\hline TSC & 0.702 & 0.441 & 0.303 & 0.707 & $0.395 * * *$ & $0.439 * * *$ & $0.402 * * *$ & $0.550 * * *$ & $0.347 * * *$ & $0.376 * * *$ & 0.664 & & \\
\hline TDD & 0.689 & 0.425 & 0.333 & 0.692 & $0.434 * * *$ & $0.370 * * *$ & $0.409 * * *$ & $0.577 * * *$ & $0.303 * * *$ & $0.434 * * *$ & $0.510 * * *$ & 0.652 & \\
\hline CLCN & 0.684 & 0.420 & 0.267 & 0.686 & $0.456 * * *$ & $0.260 * *$ & $0.357 * * *$ & $0.471 * * *$ & $0.480 * * *$ & $0.516 * * *$ & $0.434 * * *$ & $0.381 * * *$ & 0.648 \\
\hline
\end{tabular}

Nguồn: Kết quả xử lý dữ liệu khảo sát

Mặt khác, giá trị các hệ số tương quan và sai số đo lường giữa các khái niệm đều $>0$ và khác 1 . Ngoài ra, giá trị tới hạn của các sai số đo lường đều lớn hơn rất nhiều so với 1.96, nên xác suất sai lệch nhỏ hơn $1 \%$ (Schumacker \& Lomax, 2010) qua đó kết luận rằng các khái niệm nghiên cứu đều đạt giá trị phân biệt và độ tin cậy cao cũng như đạt được tính đơn hướng.

\subsubsection{Kết quả kiểm định giả thuyêt}

\section{Đánh giá chung mức độ phù hợp của mô hình}

Như đã đề cập ở trên, tác giả kiểm định giả thuyết nghiên cứu thông qua đánh giá mô hình cấu trúc tuyến tính. Vì kết quả phân phối (ở Bảng 2) của các biến quan sát có các độ lệch và độ nhọn nằm trong khoảng $[-1,+1]$ nên tác giả sử dụng ước lượng phương pháp $\mathrm{ML}$ (Muthén \& Kaplan, 1985). Theo kết quả SEM với ước lượng ML bảng dưới đây, mô hình có 361 bậc tự do, Chi bình phương/bậc tự đó đạt 1.659, CFI đạt 0.911, TLI đạt 0.9, RMSEA đạt 0.044, cho thấy mô hình cấu trúc tuyến tính là phù hợp, đủ điều kiện để phân tích.

\section{Phân tích kết quả kiểm định giả thuyết}

Dựa theo kết quả ước lượng chuẩn hóa ở Bảng 6, với mức ý nghĩa thống kê $95 \%$, các giả thuyết $\mathrm{H1}$, H6b, H7b, H8a bị bác bỏ, bởi xác suất ( $\mathrm{p}>0.005)$. Qua các hệ số ước lượng cho thấy, mối quan hệ khá cao giữa TTC (Tính tin cậy) với TSC (Tính sẵn có), từ đó cho thấy rằng tính tin cậy đối với ĐHTM sẽ có ý nghĩa lớn nếu người sử dụng cảm nhận được tính tin cậy từ đồng hồ. Tiếp theo, cũng dựa vào hệ số ước lượng, chất lượng cảm nhận sẽ tác động mạnh đối với tính dễ sử dụng hơn tính hữu dụng đối với ĐHTM. Mặt khác, tính di động chỉ có tác động ý nghĩa đối với tính hữu dụng, chứ không tác động có ý nghĩa đối với tính dễ sử dụng. Tương tự, tính sẵn có chỉ có tác động ý nghĩa đối với tính hữu dụng, chứ không tác động có ý nghĩa đối với tính dễ sử dụng. Thêm vào đó, tính hữu dụng sẽ tác động đến thái độ đối với ĐHTM hơn tính dễ sử dụng. Tính hữu dụng cũng được khẳng định tác động có ý nghĩa đến ý định sử dụng ĐHTM. Và cuối cùng, đặc tính văn hóa cũng được khẳng định tác động mạnh và có ý nghĩa đến thái độ và ý định sử dụng ĐHTM. 


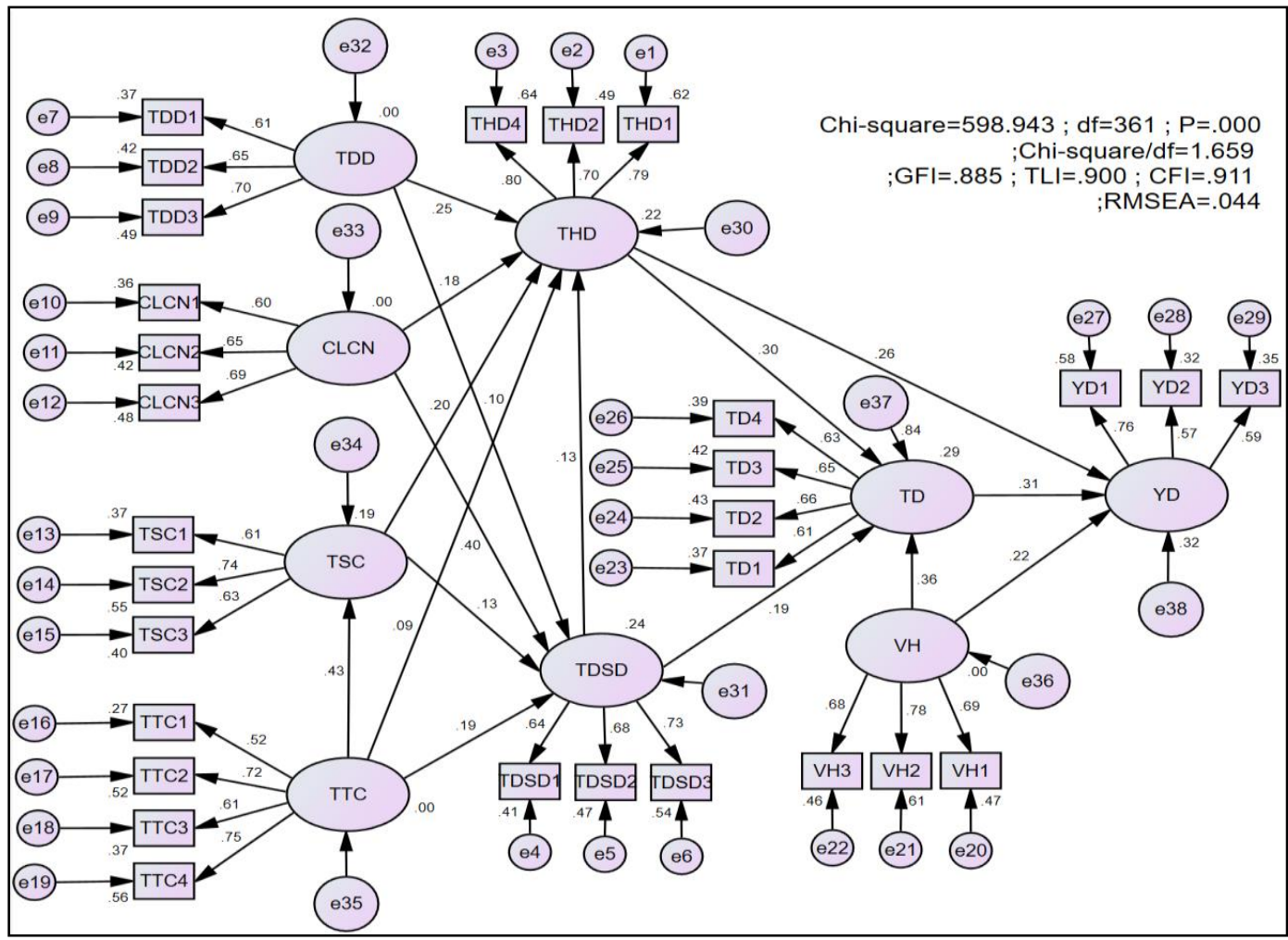

Hình 3. Kết quả mô hình cấu trúc tuyến tính chuẩn hóa

Nguồn: Kết quả xử lý dữ liệu khảo sát

\section{Bảng 6}

Kết quả ước lượng mối quan hệ giữa các giả thuyết trong mô hình chuẩn hóa

\begin{tabular}{|c|l|l|l|c|c|c|c|c|}
\hline $\begin{array}{c}\text { Giả } \\
\text { thuyết }\end{array}$ & \multicolumn{2}{|c|}{ Mối quan hệ } & $\begin{array}{c}\text { Hệ số ước } \\
\text { lượng } \\
\text { chuấn hóa }\end{array}$ & $\begin{array}{c}\text { Độ lệch } \\
\text { chuẩn } \\
\text { (S.E) }\end{array}$ & $\begin{array}{c}\text { Giá trị } \\
\text { tới hạn } \\
(\mathbf{C . R})\end{array}$ & $\begin{array}{c}\text { Xác suất } \\
\text { sai lệch } \\
(\mathbf{P})\end{array}$ & $\begin{array}{c}\text { Chấp } \\
\text { nhận/bác } \\
\text { bõ }\end{array}$ \\
\hline H8c & TSC & $\leftarrow$ & TTC & 0.434 & .099 & 4.988 & $* * *$ & Chấp nhận \\
\hline H5b & TDSD & $\leftarrow$ & CLCN & 0.396 & .092 & 4.626 & $* * *$ & Chấp nhận \\
\hline H8b & TDSD & $\leftarrow$ & TTC & 0.194 & .106 & 2.366 & .018 & Chấp nhận \\
\hline H7b & TDSD & $\leftarrow$ & TSC & 0.126 & .100 & 1.428 & .153 & Bác bỏ \\
\hline H6b & TDSD & $\leftarrow$ & TDD & 0.102 & .080 & 1.270 & .204 & Bác bỏ \\
\hline H6a & THD & $\leftarrow$ & TDD & 0.251 & .070 & 3.262 & .001 & Chấp nhận \\
\hline H5a & THD & $\leftarrow$ & CLCN & 0.181 & .082 & 2.155 & .031 & Chấp nhận \\
\hline H7a & THD & $\leftarrow$ & TSC & 0.201 & .087 & 2.388 & .017 & Chấp nhận \\
\hline H1 & THD & $\leftarrow$ & TDSD & 0.132 & .076 & 1.581 & .114 & Bác bỏ \\
\hline H8a & THD & $\leftarrow$ & TTC & 0.088 & .092 & 1.129 & .259 & Bác bỏ \\
\hline H3a & TD & $\leftarrow$ & THD & 0.297 & .061 & 3.950 & $* * *$ & Chấp nhận \\
\hline H2 & TD & $\leftarrow$ & TDSD & 0.193 & .055 & 2.565 & .010 & Chấp nhận \\
\hline
\end{tabular}




\begin{tabular}{|c|l|l|l|c|c|c|c|c|}
\hline $\begin{array}{c}\text { Giả } \\
\text { thuyết }\end{array}$ & \multicolumn{3}{|c|}{ Mối quan hệ } & $\begin{array}{c}\text { Hệ số ước } \\
\text { lượng } \\
\text { chuấn hóa }\end{array}$ & $\begin{array}{c}\text { Độ lệch } \\
\text { chuẩn } \\
\text { (S.E) }\end{array}$ & $\begin{array}{c}\text { Giá trị } \\
\text { tới hạn } \\
\text { (C.R) }\end{array}$ & $\begin{array}{c}\text { Xác suất } \\
\text { sai lệch } \\
(\mathbf{P})\end{array}$ & $\begin{array}{c}\text { Chấp } \\
\text { nhận/bác } \\
\text { bỏ }\end{array}$ \\
\hline H9a & TD & $\leftarrow$ & VH & 0.357 & .063 & 4.493 & $* * *$ & Chấp nhận \\
\hline H4 & YD & $\leftarrow$ & TD & 0.390 & .123 & 3.372 & $* * *$ & Chấp nhận \\
\hline H3b & YD & $\leftarrow$ & THD & 0.262 & .081 & 3.488 & $* * *$ & Chấp nhận \\
\hline H9b & YD & $\leftarrow$ & VH & 0.217 & .088 & 2.605 & .009 & Chấp nhận \\
\hline
\end{tabular}

Nguồn: Kết quả xử lý dữ liệu khảo sát

\section{Ước lương mô hình lý thuyết bằng Bootstrap}

Để khẳng định giá trị, sự phù hợp của mô hình lý thuyết, nhóm tác giả sử dụng ước lượng bằng phương pháp Bootstrap, với mẫu lặp lại 1,000. Theo kết quả ở Bảng 7 , cho thấy kết quả ước lượng bằng Bootstrap với kết quả ước lượng chính thức (Bằng $\mathrm{ML}$ ) có độ sai lệch rất nhỏ qua hệ số Bias và SE-Bias. Mặt khác, giá trị tuyệt đối của hệ số C.R của tất cả các ước lượng đều nhỏ hơn 1.96. Qua đó, cho thấy mô hình ước lượng Bootstrap là phù hợp, đạt độ tin cậy với 95\%.

\section{Bảng 7}

Kết quả ước lượng mối quan hệ giữa các giả thuyết trong mô hình chuẩn hóa bằng Bootstrap

\begin{tabular}{|l|l|l|c|c|c|c|c|c|}
\hline \multicolumn{2}{|c|}{ Mối quan hệ } & SE & SE-SE & $\begin{array}{c}\text { Ước lưọng } \\
\text { (Bootstrap) }\end{array}$ & Bias & SE-Bias & C.R \\
\hline TSC & $\leftarrow$ & TTC & .066 & .001 & .435 & .001 & .002 & 0.50 \\
\hline TDSD & $\leftarrow$ & CLCN & .080 & .002 & .392 & -.004 & .003 & -1.33 \\
\hline TDSD & $\leftarrow$ & TTC & .083 & .002 & .192 & -.002 & .003 & -0.67 \\
\hline TDSD & $\leftarrow$ & TSC & .093 & .002 & .129 & .003 & .003 & 1.00 \\
\hline TDSD & $\leftarrow$ & TDD & .091 & .002 & .101 & -.001 & .003 & -0.33 \\
\hline THD & $\leftarrow$ & TDD & .082 & .002 & .249 & -.002 & .003 & -0.67 \\
\hline THD & $\leftarrow$ & CLCN & .089 & .002 & .178 & -.002 & .003 & -0.67 \\
\hline THD & $\leftarrow$ & TSC & .090 & .002 & .205 & .003 & .003 & 1.00 \\
\hline THD & $\leftarrow$ & TDSD & .098 & .002 & .133 & .001 & .003 & 0.33 \\
\hline THD & $\leftarrow$ & TTC & .078 & .002 & .087 & -.001 & .002 & -0.50 \\
\hline TD & $\leftarrow$ & THD & .069 & .002 & .300 & .003 & .002 & 1.50 \\
\hline TD & $\leftarrow$ & TDSD & .079 & .002 & .193 & .000 & .003 & 0.00 \\
\hline TD & $\leftarrow$ & VH & .073 & .002 & .356 & -.002 & .002 & -1.00 \\
\hline YD & $\leftarrow$ & TD & .097 & .002 & .313 & .003 & .003 & 1.00 \\
\hline YD & $\leftarrow$ & THD & .085 & .002 & .263 & .001 & .003 & 0.50 \\
\hline YD & $\leftarrow$ & VH & .088 & .002 & .217 & -.001 & .003 & -1.33 \\
\hline
\end{tabular}

Nguồn: Kết quả xử lý dữ liệu khảo sát 


\section{Thảo luận và hàm ý quản trị từ kết quả nghiên cứu}

Mục đích của nghiên cứu này là khám phá các nhân tố tác động đến ý định sử dụng ĐHTM của người tiêu dùng Việt Nam. Trên cơ sở ứng dụng và mở rộng mô hình TAM (nhận thức tính hữu dụng và tính dễ sử dụng) và có xét đến đặc tính văn hóa của người tiêu dùng Việt Nam đối với thái độ và ý định sử dụng ĐHTM. Kết quả nghiên cứu khẳng định các giả thuyết, mô hình TAM đối với ý định sử dụng ĐHTM của người tiêu dùng Việt Nam. Kết quả nghiên cứu đem lại một số hàm ý về lý thuyết và thực tiễn cho các nhà kinh doanh đồng hồ thông minh tại thị trường Việt Nam như sau:

Thứ nhất, ý định sử dụng ĐHTM của người tiêu dùng Việt Nam trước hết chịu sự tác động của nhận thức tính hữu dụng và nhận thức tính dễ sử dụng. Kết quả này lần nữa khẳng định đề xuất của mô hình TAM (Davis, 1989, 1993) và các nghiên cứu ở các thị trường khác như nghiên cứu của Kim và Shin (2015) đối với thị trường Hàn Quốc, Dutot và các cộng sự (2019) đối với thị trường Pháp, Thái Lan và Trung Quốc. Từ đó, các doanh nghiệp kinh doanh ĐHTM tại Việt Nam cần lưu ý rằng người tiêu dùng khi sử dụng ĐHTM, họ không chỉ quan tâm đến tính hữu dụng của sản phẩm mà còn quan tâm đến khả năng sử dụng sản phâm có thuận lợi, dễ dàng không;

Thứ hai, nhận thức tính hữu dụng đối với ĐHTM có ý nghĩa lớn với thái độ và ý định sử dụng. Trong đó, nhận thức về tính dễ sử dụng có tác động nhỏ hơn đối với thái độ đối với ĐHTM. Qua đó, cho thấy người tiêu dùng Việt Nam quan tâm đến tính hữu dụng của ĐHTM trong việc lựa chọn sử dụng thiết bị công nghệ này. Kết quả nghiên cứu cũng cho thấy người tiêu dùng đánh giá cao về tính hữu dụng của ĐHTM đối với tính năng cảnh báo nguy hiểm, nâng cao sức khỏe, bên cạnh nâng cao hiệu quả công việc;

Thứ ba, kết quả nghiên cứu cũng khám phá ra rằng có mối quan hệ cao giữa tính tin cậy và tính sẵn có theo đánh giá của người tiêu dùng Việt Nam đối với ĐHTM. Đây là điểm mới, ít được quan tâm trong các nghiên cứu khác, khi nghiên cứu 2 nhân tố này tách biệt nhau. Do đó, đối với các nhà kinh doanh ĐHTM tại Việt Nam cần chú ý đến các dòng ĐHTM thỏa được mối quan hệ trên cũng như truyền thông nhấn mạnh đê người tiêu dùng yên tâm khi sử dụng;

Thứ tư, kết quả nghiên cứu khẳng định rằng đặc tính văn hóa của Việt Nam không chỉ ảnh hưởng đến thái độ mà còn ảnh hưởng đến ý định sử dụng ĐHTM. Kết quả nghiên cứu bổ sung khám phá thêm mối quan hệ giữa đặc tính văn hóa với ý định sử dụng ĐHTM, điều mà các nghiên cứu cứu trước đó chỉ khám phá ra mối quan hệ giữa đặc tính văn hóa với thái độ (Kim \& Shin, 2015) tại thị trường Hàn Quốc. Qua đó, cho thấy ĐHTM không chỉ là phương tiện theo dõi thời gian mà còn thể hiện tính thời trang, cá tính của người sử dụng. Các doanh nghiệp kinh doanh ĐHTM có thể dựa trên điều này, tạo ra các chương trình quảng cáo, truyền thông để khuếch đại, lan tỏa trào lưu theo đám đồng (đặc tính văn hóa của người tiêu dùng Việt Nam) đối với ý định sư dụng ĐHTM.

\section{Hạn chế và hướng nghiên cứu tiếp theo}

Nghiên cứu này còn một số hạn chế. Đầu tiên, nghiên cứu này thực hiện khảo sát trực tuyến và mẫu khảo sát theo phương pháp thuận tiện. Tiếp theo, đối tượng khảo sát chủ yếu các tỉnh miền đông, tây nam bộ và trung bộ. Mô hình cần được kiểm định tại các địa điểm khác của Việt Nam như các tỉnh bắc bộ và tây nguyên. Ngoài ra đối tượng khảo sát tập trung vào đối tượng sinh viên năm thứ hai trở đi và người đi làm, chưa khảo sát đối tượng học sinh, đặc biệt là học sinh phổ thông, cũng là khách hàng sử dụng ĐHTM. 


\section{Tài liệu tham khảo}

Ajzen, I. (1991). The theory of planned behavior. Organizational Behavior and Human Decision Processes, 50(2), 179-211.

Ajzen, I. (2002). Perceived behavioral control, self-efficacy, locus of control, and the theory of planned behavior 1. Journal of Applied Social Psychology, 32(4), 665-683.

Aljifri, H. A., Pons, A., \& Collins, D. (2003). Global e-commerce: A framework for understanding and overcoming the trust barrier. Information Management and Computer Security, 11(3), 130-138.

Anderson, J. C., \& Gerbing, D. W. (1988). Structural equation modeling in practice: A review and recommended two-step approach. Psychological Bulletin, 103(3), 411-423.

Bajarin, T. (2014). 3 things smartwatches need to be ready for prime time. Retrieved January 23, 2020, from http://time.com/6545/3-things-smartwatches-need-to-be-ready-for-prime-time

Cecchinato, M. E., Cox, A. L., \& Bird, J. (2015). Smartwatches: The good, the bad and the ugly? Proceedings of the 33rd Annual ACM Conference Extended Abstracts on Human Factors in Computing Systems, 2133-2138. New York, NY: ACM.

Christensson, P. (2017). Smartwatch definition. Retrieved January 20, 2020, from https://techterms.com

Davis, F. D. (1989). Perceived usefulness, perceived ease of use, and user acceptance of information technology. MIS Quarterly, 13(3), 319-340.

Davis, F. D. (1993). User acceptance of information technology: System characteristics, user perceptions and behavioral impacts. International Journal of Man-Machine Studies, 38(3), 475-487.

Dutot, V. (2015). Factors influencing Near Field Communication (NFC) adoption: An extended TAM approach. The Journal of High Technology Management Research, 26(1), 45-57.

Dutot, V., Bhatiasevi, V., \& Bellahom, N. (2019). Applying the technology acceptance model in a three-countries study of smartwatch adoption. Journal of High Technology Management Research, 30(1), 1-14.

Elbeck, M., \& Tirtiroglu, E. (2008). Qualifying purchase intentions using queueing theory. Journal of Applied Quantitative Methods, 3(2), 167-178.

Fishbein, M., \& Ajzen, I. (1975). Belief, attitude, intention and behavior: An introduction to theory and research. Boston, MA: Addison-Wesley.

Fornell, C., \& Larcker, D. F. (1981). Evaluating structural equation models with unobservable variables and measurement error. Journal of Marketing Research, 18(1), 39-50.

Gefen, D., Karahanna, E., \& Straub, D. (2003). Inexperience and experience with online stores: The importance of tam and trust. IEEE Transactions on Engineering Management, 50(3), 307-321.

Hair, J. F., Black, W. C., Babin B. J., \& Anderson R. E. (2009). Multivariate data analysis (7th ed.). Upper Saddle River, NJ: Pearson Prentice Hall.

Hair, J. F., Black, W. C., Babin B. J., \& Anderson R. E. (2014). Multivariate data analysis (7th International ed.). Harlow, UK: Pearson Education Limited. 
Hofstede, G. (1983). The cultural relativity of organizational practices and theories. Journal of International Business Studies, 14(2), 75-89.

Howard, J. A., \& Sheth, J. N. (1969). Theory of buyer behavior. New York, NY: Wiley \& Sons.

Kim, K., \& Shin, J. (2015). An acceptance model for smart watches implications for the adoption of future wearable technology. Internet Research, 25(4), 527-541.

Muthén, B., \& Kaplan, D. (1985). A comparison of some methodologies for the factor analysis of nonnormal Likert variables. British Journal of Mathematical and Statistical Psychology, $38(2), 171-180$.

Nguyen, T. D., \& Nguyen, T. T. M. (2011). Nghiên cứu khoa hoc Marketing - Úng dụng mô hình cấu trúc tuyến tính SEM [Marketing scientific research - Applying SEM]. Hanoi, Vietnam: NXB Lao Động.

Rakuten Insight. (2018). Wearables in Asia. Retrieved March 28, 2020, from https://insight.rakuten.com/ wearables-in-asia/

Reid, M., \& Yair, L. (2008). Integrating trust and computer self-efficacy with TAM: An empirical assessment of customers' acceptance of Banking Information Systems (BIS) in Jamaica. Journal of Internet Banking and Commerce, 12(3), 1-18.

Russell, J. A. (2003). Core affect and the psychological construction of emotion. Psychological Review, 110(1), 145-172.

Schumacker, R. E., \& Lomax, R. G. (2010). A beginner's guide to structural equation modeling (3rd ed.). New York, NY: Routledge.

Seppälä, P., \& Alamäki, H. (2003). Mobile learning in teacher training. Journal of Computer Assisted Learning, 19(3), 330-335.

Shin, D. H. (2012). What makes consumers use VoIP over mobile phones? Free riding or consumerization of new service. Telecommunications Policy, 36(4), 311-323.

Srivastava, S., Chandra, S., \& Theng, Y. (2010). Evaluating the role of trust in consumer adoption of mobile payment systems: An empirical analysis. Communications of the Association for Information Systems, 27(1), 561-588.

Tucker, L. R., \& Lewis, C. (1973). A reliability coefficient for maximum likelihood factor analysis. Psychometrika, 38(1), 1-10. 


\section{PHỤ LỤC}

Danh mục các biến quan sát chính thức

Nhận thức tính hĩu dụng

THD1 - ĐHTM có thể giúp tôi giải cứu và cảnh báo trong tình huống nguy hiểm

THD2 - ĐHTM có thể giúp tôi theo dõi sức khoẻ liên tục

THD3 - ĐHTM có thể giúp tôi làm được nhiều công việc (học tập) hơn

THD4 - ĐHTM có thể giúp tôi thực hiện công việc (học tập) của mình một cách hiệu quả hơn

Nhận thức tính dễ sủ dụng

TDSD1 - Tôi nhận thấy các thao tác trên ĐHTM rất dễ dàng.

TDSD2 - Tìm hiểu cách sử dụng ĐHTM đối với tôi không tốn quá nhiều nỗ lực và thời gian

TDSD3 - Tôi có thể tự tìm hiểu cách sử dụng ĐHTM một mình.

Chất lượng cảm nhận

CLCN1 - Tôi cảm thấy có thể bị thu hút bởi thiết kế của ĐHTM

CLCN2 - ĐHTM có thể kích thích tôi muốn sử dụng

CLCN3 - Tôi cảm thấy có thể hài lòng với ĐHTM (bởi chất lượng/tính năng)

Tính sã̃n có

TSC1 - ĐHTM có thể giúp tôi tìm kiếm thông tin và dịch vụ tôi cần.

TSC2 - ĐHTM có thể chủ động cung cấp thông tin nên khiến tôi cảm giác thông tin luôn được cung cấp vào đúng lúc

TSC3 - ĐHTM có thể cung cấp những thông tin chính xác và nhanh chóng

Tính tin cậy

TTC1 - Chính sách của các hãng ĐHTM về việc sẽ sử dụng thông tin cá nhân của tôi có thể khiến tôi cảm thấy sản phẩm công nghệ này đáng tin cậy

TTC2 - Chính sách của các hãng ĐHTM về cách họ sẽ chia sẻ thông tin cá nhân của tôi với bên thứ 3 có thể khiến tôi cảm thấy sản phẩm công nghệ này đáng tin cậy

TTC3 - Tôi có thể cảm thấy sản phẩm công nghệ này đáng tin cậy vì thông tin cá nhân của tôi luôn được cập nhật đầy đủ và chính xác

TTC4 - Các quy tắc bảo mật dữ liệu của hãng sản xuất có thể khiến tôi cảm thấy tự tin hơn về việc sử dụng ĐHTM

Tính di động

TDD1 - ĐHTM có thể dễ dàng mang theo bên người

TDD2 - Tôi có thể sử dụng ĐHTM ở bất kỳ đâu

TDD3 - Tôi có thể thích sử dụng ĐHTM khi tôi đang di chuyển từ nơi này sang một nơi khác

Đặc tính văn hóa

VH1- ĐHTM khiến tôi trở nên khác biệt so với mọi người. 
VH2 - ĐHTM khiến tôi trở nên nổi bật so với mọi người.

VH3 - Những người sử dụng ĐHTM thường là những người đặc biệt

Thái độ

TD1 - Sử dụng ĐHTM có thể là một ý tưởng tuyệt vời

TD2 - Tôi có thể thích sử dụng ĐHTM

TD3 - Sử dụng ĐHTM có thể rất có ích

TD4 - Sử dụng ĐHTM có thể rất hiệu quả

Ý định sủ dụng

YD1- Tôi hy vọng sử dụng ĐHTM trong thời gian tới.

YD2 - Tôi đã lên kế hoạch cho việc sử dụng ĐHTM.

YD3 - ĐHTM là lựa chọn ưu tiên sử dụng của tôi trong thời gian tới 